\title{
Towards a Probe Design Framework
}

\author{
Eleanor Chin Derix \\ Interaction Design + Human Practice Lab \\ University Technology Sydney \\ Sydney, NSW Australia \\ eleanor.derix@gmail.com
}

\author{
Tuck Wah Leong \\ Interaction Design + Human Practice Lab \\ University Technology Sydney \\ Sydney, NSW Australia \\ tuckwah.leong@uts.edu.au
}

\begin{abstract}
Since their introduction, probes have been widely used in HCI. Despite this, there have not been much reflections and discussions about the design thinking behind their creation and use. There is also a lack of actionable guidance on designing and using probes. This lack may have contributed to some concerns that the method has been misinterpreted and misunderstood. We reviewed HCI literature surrounding probes and found one of the few papers that offers a nascent framework for probe design and use. We used it to guide the design of a collection of probes and reflected on the framework's usefulness. We extend this framework by offering a more useful way of visualizing and working with probe design properties. We also provide further clarity and advice on how others may think and approach the design and use of probes more effectively, especially those turning to probes for the first time.
\end{abstract}

\section{CCS CONCEPTS}

- Human-centered computing $\rightarrow$ HCI design and evaluation methods

\section{KEYWORDS}

probes; design; framework; guidance

\section{ACM Reference format:}

Eleanor Chin Derix and Tuck Wah Leong. 2019. Towards a Probe Design Framework. In Proceedings of ACM OZCHI Conference (OZCHI'19). ACM, New York, NY, USA, 2 pages. https://doi.org/10.1145/3369457.3369467

\section{Introduction}

This is a methods paper that contributes to current understandings of how probes can be designed more thoughtfully and strategically, to support user research in HCI. More specifically, it describes how

Permission to make digital or hard copies of all or part of this work for personal or classroom use is granted without fee provided that copies are not made or distributed for profit or commercial advantage and that copies bear this notice and the full citation on the first page. Copyrights for components of this work owned by others than ACM must be honored. Abstracting with credit is permitted. To copy otherwise, or republish, to post on servers or to redistribute to lists, requires prior specific permission and/or a fee. Request permissions from Permissions@acm.org. we utilized Wallace et al.'s [21] framework that guides probe design and use to help inform our decision-making when developing a set of probes of our own. We reflect on our probes design process and how our research participants used the probes to ascertain the usefulness and effectiveness of this framework. This leads to suggestions and insights as to how this framework could be extended and tested, so as to be more helpful to HCI researchers. This contribution is particularly valuable in supporting (budding) researchers and designers contemplating probes as a method; offering a more structured and strategic way to think about the decisions taken when designing and using probes.

After all, these decisions can impact how deeply participants engage with our probes, the quality of their responses, and their overall sensemaking of these designed objects of inquiry.

The need to develop a set of probes came from our research, which explores the complex experiences associated with family technology use. In particular, we were interested in capturing the different individual perspectives held by parents within the same family [10]. We planned to supplement a series of in-home interviews with probes as a means of encouraging participants to reflect on aspects of routine technology use that are often overlooked within the messiness of everyday family life. When reviewing the literature on probes, we found many publications describing probes, but that only Wallace et al.'s [21] paper went some way to providing comprehensive 'guidance', in the form of a framework. So, we were interested in exploring the usefulness of this framework to guide us in designing the probes for our research project.

Our review of related work will unpack some of the debate and concerns around probes, particularly around the lack of clarity about the method itself. We also discuss the availability of design guidance offered within the literature on probes, in particular the one presented by Wallace et al. [21]. We then describe how we operationalized this framework to guide the design and use of a set of probes. First, distilling the framework: outlining the key design properties of probes and the decisions that affect them. Second, putting the distilled framework to use as a guide to design and use of three probes of our own. The 'findings' section will be our reflections on the effectiveness of the framework to guide the design of a probe collection. We also discuss the framework's utility by considering how participants responded to using these probes. Finally, by reflecting on what we learned by using the 
framework in this way, we suggest refinements, extensions, and ways that the framework could be adapted and tested in future.

\section{Related Work}

Since their conception by Gaver et al. [11] probes have become a well-established approach to understanding users, their behaviours, and use of technologies [6]. However, amidst this enthusiastic uptake of the method within HCI and design, concerns have been raised about the misinterpretation and misappropriation of probes. In particular, Boehner et al. [5] suggest that this may be due to a lack of clarity on the method itself, with accounts of probe use tending to gloss over details of how they were designed. Some researchers have attempted to add clarity to the method by discussing what probes are [5] and what they do [2, 14]. Attempts have been made to catalogue different kinds of probes $[13,18]$, for instance by topic of interest (e.g. domestic probes, urban probes etc.), desired result (e.g. empathy probes, value probes etc.) or new approaches to using probes (e.g. mobile probes, technology probes etc.) [5]. Another effort to provide clarity has been to try and determine what these different probes have in common (e.g. probes inspire, probes create fragments, probes provoke...etc.) [14]. Despite these efforts, clear guidance on how to actually design probes remains elusive.

\subsection{Existing Probe Design Guidance}

Most publications involving the use of probes discuss what probes are and what probes do, furthering Gaver et al.'s [12] original definition of cultural probes as "collections of evocative tasks meant to elicit inspirational responses from people". Detailed guidance on how to design probes is limited. Instead, advice centers on how to approach the probe design process. For instance, in their outline of the probe design process, Hemmings et al. [15] discuss various skills required by those wishing to adopt the method (e.g. idea generation, graphic design, model etc.) and list the phases involved (e.g. recruitment, assembling probes, deploying probes, retrieving probes etc.). However, while they highlight the need for design skills and for team discussions to generate probe ideas, they neglect to include a probe design phase from their schedule which moves straight from "Selecting Volunteers" to "Assembling Domestic Probes". The tendency to gloss over the design thinking behind probes is common in probe literature.

We found guidance on how to think about probes. For example, Graham et al. [13] define common probe features (e.g. capture artefacts, making the invisible visible, participant as expert etc.) and their effects (e.g. humanize, create fragments etc.). Guidance is also offered on how to generate the questions being asked through the use of probes. For instance, Mattelmaki's [18] introduction to the method suggests considering participation, before designing probes (e.g. "Who is your user?" "How long will people be involved?" etc.). In addition, The Interaction Design Studio [19] offer approaches to prompt the ideation of probe concepts. (e.g. "use analogies", "ask obliquely-related questions" etc.) and provide examples of probe tools. We acknowledge that these attempts add clarity to the method. However, we still lacked more explicit/detailed guidance about the design decisions required to develop a probe collection.

To be fair, there are a few authors who describe the thinking behind their probe designs in more detail. For example, Tsai et al. [20] describe their rationale for designing Memory Probes; balancing three sets of probe properties ("familiarity-strangeness", "definiteness-ambiguity" and "objective-subjective"). Boucher et al. [6] also discuss probe properties (e.g. "simple and easy", "openended", "playful" and "absurd" etc.) when introducing a novel probe tool, TaskCam. However, while these reports provide insights and details into decisions taken to designing probes, these efforts are not aimed at providing general advice or guide for effective probe design decisions. These occasional glimpses into differing ways of thinking and also talking about the design properties of probes further highlight a need for clearer, more consistent guidance. One exception is a paper by Wallace et al. [21], which provides a systematic reflection on probe design decisions. One of its explicit aims is an "attempt to address the identified lacuna" - which is "the lack of accounts that describe in detail the design of probes and their use with participants". Some have argued that this lacuna is one of the reasons why the method has been often misinterpreted and proved elusive to many.

\subsection{Wallace et al.'s Framework}

In Making Design Probes Work, Wallace et al. [21] offer what they call "a framework for probe design and use" based on detailed descriptions of the design of probes and their use with participants. This salient guide, which we will refer to in this paper as 'the framework' focuses explicitly on the design decisions required to develop probes. It is a summary of learnings from their projects spanning over a decade involving the design and use of probes.

The framework in this paper consists of two types of guidance. The first is a lexicon of probe design properties; which can be used in probe design to provide "scaffolds for response". This section also offers guidance as to how design decisions can affect particular design properties and, in turn, participant engagement and response. The second type of guidance offered in this paper is less prescriptive. It relates to supporting "relationships and reciprocity" and includes ways to best consider and involve participants when designing probe studies. To the best of our knowledge, nobody has explicitly described putting this framework to use. So, our initial aim was to ascertain the effectiveness of this framework as a guide to design a set of probes deployed in an empirical study. As mentioned earlier, this was part of our research into parents' experiences of family technology use.

\section{Method}

We quickly realized that operationalizing Wallace et al.'s framework to design our probes was not a straightforward exercise. They have provided some resources and general advice but we had to first distill the various elements to make it useful. 


\subsection{Distilling the Framework}

As we mentioned earlier, one set of guidance from the framework describes four probe design properties. First, openness/boundedness relates to how clear or vague a participant finds the question being asked by a probe as well as what is required to complete it. Second, materiality relates to the physicality of the probe tool (or artefact) that might help embody the question being asked by a probe, or encourage a particular type of response from participants. Finally, pace and challenge relates to the time and effort required to complete a probe.

Openness/Boundedness: The framework explains this property by describing the design of the probe Self Tree. Participants were asked to write about people in their lives on a series of oval, locketlike paper discs. This example shows how the openness or boundedness of a particular probe can be determined by both the physical dimensions of a probe tool and conceptual decisions to define a probe task. For instance, the openness of the question asked by Self Tree is balanced by the choice to use small paper discs that restrict the amount that can be written.

Materiality: The framework describes how material choices, and decisions around the shape, style and finished appearance contribute to the materiality of a probe. The examples used to describe this design property reference relevant objects in order to invoke an intended response from participants. The use of physical metaphor is demonstrated through the example of Home probe, intended to capture participants' sense of home and designed as a cardboard structure in the form of a house. More subtle references are shown through the example of Pillow probe and Self Tree. The former aims to invoke a sense of intimacy by asking participants to write on a pillow, while the latter aims to suggest preciousness by taking the form of jewelry.

Pace: The framework describes how probes can be designed to encourage faster responses from participants. In particular, they describe breaking a probe task up into smaller chunks that participants perceive as being more completable. The example of Top Trumps probe is described, in which the request for participants to describe objects that are significant to them is broken down into smaller activities by using six playing cards.

Challenge: The framework highlights the need to offer probes that offer space for deeper reflection on certain topics or to tease out issues that are more difficult to express. It describes how probes designed to do this often presents participants with higher levels of challenge. As an example, it uses the design of the probe Communication Fairytale, a short storybook that creates an imaginary scenario and enables participants to express complex ideas, such as how they feel loved, as one of the characters. These more imaginary scenarios remove the restraints of what is possible and instead afford freedom from inhibitions and realities. This promotes participants reflecting from fresh perspectives.

\begin{tabular}{|c|c|}
\hline $\begin{array}{l}\text { Probe Design } \\
\text { Property }\end{array}$ & Design Decision \\
\hline \multirow[t]{2}{*}{$\begin{array}{l}\text { Openness/ } \\
\text { Boundedness }\end{array}$} & $\begin{array}{l}\text { Scale: e.g. provide small vs. large physical } \\
\text { boundaries for response }\end{array}$ \\
\hline & $\begin{array}{l}\text { Context: e.g. provide real vs. imagined } \\
\text { scenario }\end{array}$ \\
\hline \multirow[t]{3}{*}{ Materiality } & $\begin{array}{l}\text { Materials: e.g. use novel vs. familiar } \\
\text { materials }\end{array}$ \\
\hline & $\begin{array}{l}\text { Shape and Style: e.g. reference familiar } \\
\text { objects or ideas, use physical metaphor }\end{array}$ \\
\hline & $\begin{array}{l}\text { Aesthetic: e.g. create rough vs. polished } \\
\text { finished appearance }\end{array}$ \\
\hline \multirow[t]{3}{*}{ Pace } & $\begin{array}{l}\text { Speed: e.g. offer the opportunity for fast vs. } \\
\text { slow response }\end{array}$ \\
\hline & $\begin{array}{l}\text { Duration: e.g. offer long vs. short time within } \\
\text { which to respond }\end{array}$ \\
\hline & $\begin{array}{l}\text { Frequency: e.g. offer the opportunity for } \\
\text { single vs. multiple responses over time }\end{array}$ \\
\hline \multirow[t]{2}{*}{ Challenge } & $\begin{array}{l}\text { Level of Commitment: e.g. encourage light vs. } \\
\text { greater effort }\end{array}$ \\
\hline & $\begin{array}{l}\text { Level of Creativity: e.g. encourage factual } \\
\text { responses vs. use of imagination }\end{array}$ \\
\hline
\end{tabular}

Table 1. Distilling the framework: probe design properties

By discussing their own probes, Wallace et al. exemplify how different design properties can be put to use. So, we had to first analyse and interpret the various design guidance in relation to the specific probes described. We then distilled this set of guidance into a more structured and more generally applicable

set of design direction, by mapping each of the probe design properties to corresponding design decisions (see Table 1). As we did this, we noticed that probe design properties can relate to probe tools (i.e. artefacts) and/or probe tasks (i.e. activities). Materiality tends to relate to the artefact, while pace and challenge tend to relate to the task and openness/boundedness often relates to both.

\subsection{Using the Framework}

After analyzing and distilling the guidance from the framework, we then put it to use. In general, this meant adopting the approach suggested. And when we were ready to design our probes, we used the information from Table 1 to guide our design decisions. Next, we describe the process chronologically.

3.2.1 Investment and Trust: building relationships. Following Wallace et al. [21], we began with considerations for investment and trust. This means, prior to designing the probes, researchers should first build an understanding of the participants and their context to inform the design of probes. In our project, we held a workshop with parents to gain initial insights into their experiences of family technology use [10]. We then used these insights to design a collection of probes that would be given to eight sets of parents to use within a two-week study. We planned to introduce our probes to each set of parents during an in-home 'opening' interview on Day 1. Completed probes would be collected 10-12 days later and reviewed to inform 'closing' 
interviews planned for Day 14. Due to the space constraints of this paper we are unable to elaborate on the ideation process of the study and focus instead on how we used the framework to guide the design of a collection of three individual probes.

\subsubsection{Design Properties: supporting thinking for probe}

designs. We used the information in Table 1 to guide the design of each of our three probes. We used the four probe design properties; openness/boundedness, materiality, pace, and challenge to systematically explore different possible probe designs. We also went back to the examples provided in the framework to find inspiration and ideas for tangible alternatives. We now describe each of our three probes and explain how their design was guided by the framework's probe properties.

\section{Probe 1: Family Experience Jar.}

We wanted a probe that would serve as an icebreaker by encouraging participants to offer quick, regular responses and to reflect on their experiences throughout the study. We designed it as an extension of a diary, inspired by Andell et al.'s [18] stressrelaxation container. Each set of parents are given a large clear glass jar and asked to fill it with handwritten notes that log their experiences of family technology use (Fig. 1 - top). Three colours of 'post-it' style notes are provided: pink for positive experiences, blue for negative experiences and yellow for neutral or mixed experiences. We hoped that this icebreaker probe would offer participants a simple entry point into our probe collection, as recommended within the framework.

Openness/Boundedness: Since we intended Family Experience Jar to serve as an icebreaker, we kept both the concept of the question being asked and the physicality of completing the task bounded. The task requires little imagination or creativity to complete. The instructions are simple, and a reminder is written on the side of the jar. Providing small 'post-it' style notes limits the space on which to write about each experience. In contrast, the large number of notes we provided, and large size of the jar convey to participants that while we ask for at least one contribution per day, many contributions are welcome, if not expected.

Materiality: We intended for Family Experience Jar to encourage both parents within a family to offer their thoughts and feelings on experiences of family technology use. We understood that these experiences could be both overlooked and contentious. We hoped that the final appearance of the jar would remind participants of family swear jars and piggy banks. We chose clear glass jars usually bought as a decorative homeware item or vase in the hope that participants would position them in visible locations in their homes. This visibility might serve to remind participants to add contributions more regularly. The jar had a cardboard lid with a small slot cut into it. Notes must be folded in order to be fit through this opening. The lid was attached to the jar with glue so once inserted, notes could not be removed. This prevented the details of the notes being read by family members. We hoped that the privacy this affords would also encourage curiosity and further participation.

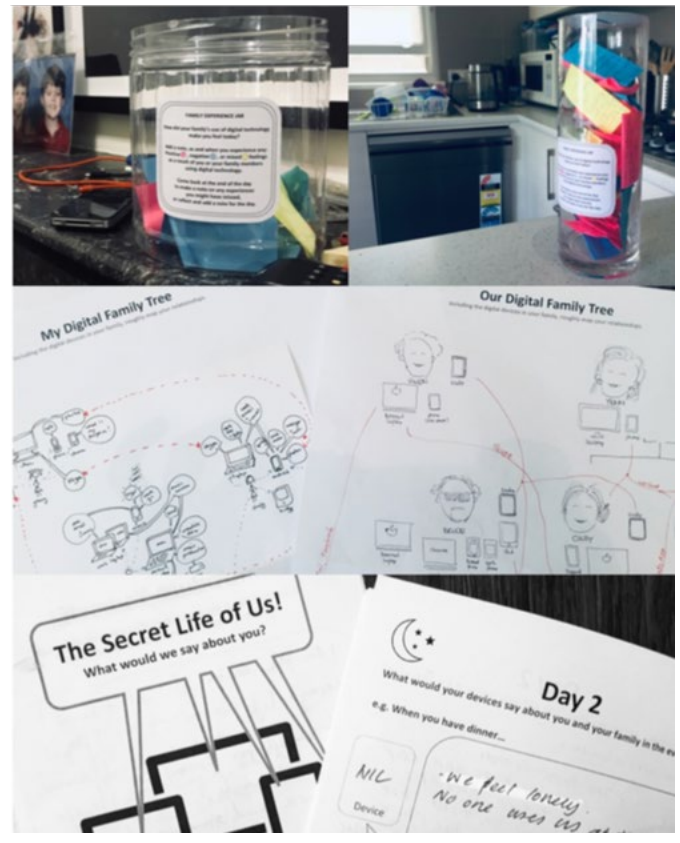

Figure 1. Using the framework to design probes. Probe 1. Family Experience Jar (top) Probe 2. Digital Family Tree (middle) Probe 3. Device Journal (bottom)

By choosing jars made of clear glass, participants could see contributions amassing over time. The visible colour of the notes inside the jar would provide an 'at-a-glance' idea of the types of experiences that had been logged. We hoped this might generate

curiosity as to what other family members have contributed; encouraging reflection and further participation. We also anticipated that the visible empty space would promote more participation.

Pace: We hoped that by compartmentalizing this 'diary' task into fast-paced, high-frequency note-taking would keep participants mindful of family technology use throughout the study. We asked participants to submit at least one note per day and invited them to make additional contributions as-and-when such experiences occur would. However, it is entirely possible for them to introduce their own flexibility with this task and add notes to the jar retrospectively. We also hoped that participants would find the physical act of selecting, writing and contributing notes to the steadily filling jar more rewarding and compelling than simply completing diary entries.

Since Family Experience Jar is intended as an icebreaker probe, we designed the task to be light-weight, requesting factual information about the realities of everyday life. It does not require much time, creativity or deep reflection. We did ask participants not to discuss contributions with other family members as we hoped that this 
element of secrecy might introduce a sense of competition and make the activity feel more playful than completing a two-week diary.

Probe 2: Digital Family Tree. We wanted this probe to help transition participants from the icebreaker task to a task that requires deeper reflection. We designed a mapping exercise in which participants create a family tree that also included the digital technologies used in everyday family life (Fig. 1 - middle). We asked each parent to complete an individual family tree during the first week of the study. During the second week we asked that each set of parents compare their individual family trees and collaborate to create a joint family tree. We hoped this probe would encourage participants to think about the role that technology plays within their family and provide overviews of the different ways in which each parent perceived technology to be incorporated within family life.

Openness/Boundedness: We provided participants with blank paper templates on which to complete this task; individual templates for the first part of the task and a shared template for the second. The minimalistic design of templates was intended to offer participants the freedom to interpret this open-ended task. We chose to use A3 sized paper hoping that it would invite selfexpression and creativity yet provide clear boundaries to convey a sense of easy completability.

When we piloted the use of this probe, we realized that more cautious participants might benefit from extra scaffolding to help explain the task and encourage creative-thinking. To do this, we prepared an example of a completed Digital Family Tree to show participants when explaining the probe activity. We were more interested in how participants interpreted this probe than in accurately recording their technology use, so took this example away once participants confirmed that our instructions. This also removed any temptation to follow our example too closely.

Materiality: When preparing our example of a completed Digital Family Tree, we tried to follow the 'typical' style of family trees and hoped participants would be especially familiar with this given the current popularity of services such as Ancestry.com. We attempted to keep our example unrefined in appearance to remove any concerns that participants' might have over the level of artistic talent expected from them.

Pace: We offered participants flexibility over when to complete this probe. We slowed the pace of this probe by asking participants to leave time between completing the individual task and collaborating on their joint family tree. We hoped that this lower pace would encourage reflection.

Challenge: We designed this probe to demand a certain level of creative thinking and imagination from participants, which we hoped would provide them fresh ways of thinking. We were inspired by Wensveen's [22] use anthropomorphism to design probes that prompt imaginative responses from participants and
Battarbee et al.'s [1] probe design that encourages creative thinking by asking participants to represent domestic appliances with animals. We hoped that using the familiar notion of family trees as a physical metaphor to pose our question would support the challenge presented by this probe.

An additional challenge presented by this probe was in asking participants to compare their individual responses and to collaborate to complete a shared family tree. This demanded extra commitment and introduced the need for communication, negotiation and collaboration. We asked participants to make a note of any difficulties they encountered to help surface insights into how parents manage their differing perspectives.

Probe 3: Device Journal: 'The Secret Life of Us'

Aspects of family technology use are often habitual and overlooked. Some are uncomfortable or even socially undesirable. We hoped that the use of this probe would provoke unexpected responses from participants by prompting them to reflect from a different point of view. To did this we designed a comic book called 'The Secret Life of Us', in which the characters are the digital technologies used within everyday family life (Fig. 1 - bottom). This probe inverts the traditional diary by asking participants not to write about their own experiences, but to imagine how their devices experience family life and to journal them in the comic over the course of two days. We were inspired to design this probe by reading about the probe Communication Fairytale in the framework.

Openness/ Boundedness: We introduced an imagined context and used anthropomorphism to make the familiar strange. This is because we hoped to prompt participants to reflect on aspects of technology that usually go unnoticed, or aspects that they are less inclined to share with researchers, such as less socially desirable contexts. As with Communication Fairytale, we hoped that creating an imagined scenario would enable participants to remove themselves from the constraints of reality and to express complex ideas as a character in a story. We balanced the openness of the ideas introduced by this probe by designing it as a (literally) bound A5 comic. By using a series of empty speech bubbles to divide each page we hoped the task would seem easily understandable and importantly, completable.

Materiality: We hoped that the compact, playful comic design would make this probe seem approachable, despite it introducing unfamiliar ideas. We hoped the use of a cartoon style would encourages participants to respond by using their imagination and creative thinking. In particular, we used device icons and speech bubbles to remind participants that we wanted them to give their technological devices an imagined voice. The design of this comic book was guided by the way Wallace et al. describe their probe Communication Fairytale as providing participants with a novel way of thinking and expressing themselves.

Pace: The aim of this probe is to provoke participants to shift their perspective and promote deeper reflections on the topic. We slowed 
the pace of Device Journal by asking participants to focus on this activity, adding at least four entries per day, and completing it over a period of two days. We hoped that the second day of journaling would encourage participants to recognize a wider range of experiences and any repetition. Participants are given the freedom to complete this journal over any two days during the study.

Challenge: This probe demands a high level of imagination and creative thinking and we hoped it would elicit deeper reflection by furthering the imagined context introduced by Probe 2 . A relatively high level of commitment is required from participants during the two days on which they complete this. First, we ask them to introduce their character (the imagined character of a particular technology) and to describe themselves and their families as they imagine their character would. Then we ask them to make regular journal entries that describe the imagined experiences of their character throughout the day.

When we piloted this probe, we realized that, as with probe 2 , our participants might benefit from additional scaffolding given the levels of imagination that this task demands. We chose to support our participants in this way by providing a link to an audio clip of 'Everything Is Alive'[7], a podcast series of fictional interviews with personified everyday objects, played by actors.

3.2.2 Reciprocity and Communication: probes as a collection. Finally, the framework encouraged us to design our probes as a collection. The rationale is that probe collections should offer participants a range channels for different kinds, types and ways to respond and reflect, to foster reciprocity and communication in the researcher-participant relationship. Our approach to designing probes was to design our three individual probes in parallel, and stepping back regularly to gauge how the individual probes complement and support each other. We were also aware that altering the design of one probe might require changes to the design of another. This also means using the different design properties (Table 1) to help vary the probes within the collection.

We found it helpful to use linear scales to represent the probe design properties (i.e., openness/boundedness, pace and challenge) as shown in Figure 2. Comparing the properties of our probes in this way helped us visualize the different role that each probe would serve within the collection. We could see that the relative

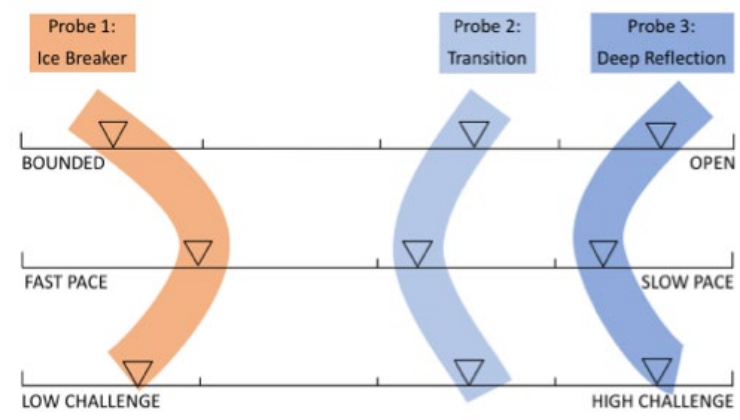

boundedness, fast-pace and low-level challenge of probe 1 would contribute to its role as an icebreaker. Meanwhile, the openness,

Figure 2. Using linear scales to visualize how three design properties vary across a probe collection

slower pace and higher challenge of probe 2 would help it transition participants towards probe 3 . We hoped the slowness, great openness and high challenge presented by this probe would enable it to encourage deep reflection from participants. We discuss the utility of these linear scales in guiding the design and use of our probes in greater detail in our findings.

\section{Findings: Usefulness of the Framework}

Probes are artefacts for inquiry, designed to be used in a bidirectional way to facilitate conversations between researchers and their participants. Thus, our findings will first reflect on the framework's utility to guide our design of our probes, and how our participants responded to these probes.

\subsection{Reflections on Probe Design}

We found that Wallace et al.'s framework useful because it provided a structured way to think about probe design and design decisions. It foregrounds the need to consider our relationships and interactions with our participants, prompts us to consider the design properties not as binary states but as properties along a continuum, as well as guided the planning, thinking and design of a varied probe collection. However, there were also parts of the framework that we found ambiguous.

4.1.1 A Structured Way to Define Probe Design Properties. The framework introduces a lexicon of four design properties openness/bounded, materiality, pace and challenge with tangible examples that helped us to better understand how to use the properties. This lexicon provided us with a clear and structured way to consider, plan and think when designing our probes. The lexicon also gave the research team a consistent terminology to talk about the probes as well as reducing potential misunderstandings.

\subsubsection{A Structured Way to Take Design Decisions. The} framework also provides a structured way to consider how different probe design properties are affected by different kinds of design decisions e.g. scale, style, aesthetic etc. This enabled us to reflect and modify the design properties of our probes in a more measured way. For example, we originally thought of probe 1 as a two-week paper experience diary. However, we anticipated that our participants would perceive this to be a heavy commitment, given how busy they had described family life to be during the preliminary workshop. We tried to reduce this apparent commitment through the design of Family Experience Jar. We hoped that participants would perceive the task of making short notes and collecting them in a jar to be less demanding.

4.1.3 Prompts Consideration of Design Properties as Continuous. We found it helpful to consider design properties as 
continuous, rather than as binary states. When describing the property openness/boundedness, the framework suggests taking design decisions that "offer a participant both openness to share whatever she feels appropriate and clear boundaries to respond within". We adapted this advice by visualizing this balancing act by means of a linear scale ranging from bound to open. As we explored with design decisions, we found it helpful to slide the relative position of a particular probe along this continuous scale. For instance, we could slide it from more open to more bound by reducing the size of a probe or slide it from more bound to more open by introducing an imagined scenario. We found it helpful to visualize the three properties in this way; openness/boundedness, pace (ranging from fast to slow) and challenge (ranging from low to high). On the other hand, we found that it is not meaningful to visualize the property materiality in this way since choices such as material, shape and style are distinct rather than continuous.

\subsubsection{Helps Guide the Design of a Varied Probe Collection.} Besides providing helpful guidance on the design of individual probes, the framework is especially effective at steering the design of varied probe collections. In particular, when we used the scales to compare openness/boundedness, pace and challenge (see Fig. 1). We realized that these properties can be used to distinguish each probe within a collection; Probe 1 (Family Experience Jar) serves as an ice-breaker, Probe 2 (Digital Family Tree) as a transition to reflection and Probe 3 (Device Journal) as a source of deep reflection. This realization helped us to ensure a collection of distinct probes that support and complement each other.

While the framework was useful, there were also aspects that were ambiguous. First, the connection between probe design properties pace and challenge, second, uncertainty over the effects of certain design decisions and finally, general difficulty in translating the second section of the framework.

4.1.5 Areas of Ambiguity. Wallace et al. discuss the design properties pace and challenge together which we found rather ambiguous both when translating the framework, and when considering the design of our own probes. These two properties may often relate to each another, however they can be affected by different design decisions. After all, it is possible that both fast and slow-paced probes could be designed to be challenging. Therefore, we chose to separate these two probe design properties in our distilled version of the framework.

Another area of ambiguity was when we tried to map the design property of materiality. Several choices that are said to affect materiality were also found to affect openness/boundedness, pace and challenge. For instance, while the use of physical metaphor is described as affecting materiality, it is also shown to affect challenge and openness/boundedness. We found that this introduced uncertainty and hesitation when distilling the framework.

The framework's lexicon of probe properties was useful, especially once we distilled it into a more usable format (Table 1). Examples of actual probes that exemplified particular design properties were very helpful to understanding how these properties could be used. It helped to clearly explain what and how we could do when designing probes. However, the framework's general advice on how to approach probes was less accessible and helpful. Understandably, this could not be as prescriptive as the probe properties. Nevertheless, we were able to interpret and heed certain advice to inform our design process. For example, we invested in time to understand our participants before beginning probe design by holding a preliminary workshop.

\subsection{Reflections on Probe Use}

The framework provided useful guidance on how to design and use our probes. Our participants were able to use the probes successfully and engage with it in the way we planned. For example, they were able to offer varying levels of responses - from quick responses to our icebreaker probe and to more reflective responses with the other probes.

4.2.1 Supporting Engaging, Quick and Easy Responses. The framework provides guidance on how to offer participants fast, light-weight probes that can serve as ice-breakers. It recommends using these probes to act as a point of entry prior to more challenging probes. We designed Probe 1 Family Experience Jar to serve as an icebreaker and encourage regular, swift, direct, physical responses. Our material and aesthetic choices had helped to ensure that this intended role was accomplished. When we first presented the three probes during opening interviews we noticed that almost all our participants immediately gravitated towards the jar. Later, when we visited our participants' homes to collect the completed probes, we observed that, as intended, jars had been placed in prominent positions such as on kitchen worktops, dining tables etc. Then when we reviewed completed probes we found our participants' responses to Family Experience Jar were the most consistent and comprehensive across all families.

When we asked participants to reflect on their overall experience of using the probes during closing interviews, most of them refer to this probe, and in particular reference the visibility of the colored notes. It appears that the transparent glass jars provided participants a view of the colored post-it notes; a visual representation of their experiences. This seems to allow them to more easily reflect and articulate their experiences easily.

4.2.2 Supporting Creative and Reflective Responses. The framework offers guidance on how to explore more difficult phenomenon by providing participants the opportunity to reflect deeper through the probes we design. For example, it suggests using tasks with a slower pace or introducing imagined contexts. When compared to Probe 1, these probes required more creativity and imagination to complete.

When we reviewed the completed probes, we found that our participants understood the task of Probe 2 (Digital Family Tree). This probe asks participants to map relationships between family 
members and their digital technologies. The task was designed to give participants the freedom to interpret the task in their own way. We saw this in the variety of response we received. For example, some showed which family members used which devices, some depicted the technologies used to connect family members and others chose to represent family members who they felt used a lot of technology by drawing a device instead of a person. The metaphor of family trees was easily understood and this probe productively facilitated fresh ways of thinking by our participants. During our discussions, they often became animated as they explained and elaborated on their creations.

Probe 3 (Device Journal) demanded the highest levels of imagination and creative-thinking. It was also designed to promote deeper reflection. It asks participants to write an imaginary journal of how their digital technologies might experience their home. When we reviewed responses, we realized that several participants had struggled with its open, slow and challenging nature. Some participants had not completed all the speech bubbles in the comic book. Some made very brief entries. Others wrote about their own experiences rather than the imagined experiences of their digital tech.

During our interviews, we discovered that most of these participants had not listened to the short audio clip that we had directed them to, to support this task. This highlighted the need to find suitable ways to scaffold probe tasks that are more challenging. Despite this, this probe inspired the most interesting and meaningful conversations during our interviews. Even participants who had struggled to complete the task could be prompted to reflect more deeply on their relationships with technology as we reviewed this probe together. These productive conversations reminded us of the importance of offering participants the freedom to not respond and to see this as a creative act in itself, as highlighted in the framework.

\subsubsection{Supporting Varying Levels of Reflection and}

Realizations. The use of a varied collection of probes allowed participants to offer a range of responses about the phenomenon of interest. We designed our probes to vary widely in both thematic context and the types of activity. Regular tasks that require short bursts of reporting, tasks that require reflection about self and others, and finally, task that require greater imagination and creativity.

When we spoke to our participants about the probes, they described how the experience of completing this range of different probes had revealed aspects about their family's technology use that they found interesting, surprising and sometimes undesirable. They explained how the activities had provided an opportunity for them to 'take stock' of their situation and that this had allowed them to make discoveries about family life, their family members and themselves.
"It enabled me to reflect on all those negative things (laughs). How much conflict there is with my son and my daughter. I wasn't aware how much that was taking up my energy I guess...

I am surprised at (my wife's) self-opinion on her devices cos she's actually on the phone a lot and she doesn't think that she is. So, I was surprised by that and I guess doing these (probe) activities gave me a legitimate lens to have a look at that... I guess I had never really tied these automatic habits, like picking up my phone, to an emotional motivation." (P9)

Some participants went further and concluded that the process of completing the probes had prompted them to consider actually making changes to their lives and their family.

"It made me really think about how to manage our time with the devices. I have actually thought about a once a month devicefree day for the whole family...to be all together on a Saturday or Sunday." (P2)

Most of our participants thanked us for the probes. They commented that the probes had provided them with an opportunity to think about not only their individual experiences but to be led to consider their family experiences more holistically and from different perspectives. This perhaps responded to the framework's recommendation for designing probes that can offer participants some degree of personal benefit during and after use.

\section{Discussion}

As our findings highlight, Wallace et al.'s framework indeed fills a void within HCI by offering us useful and actionable guidance on probe design. It does this by offering generalizable probe design properties and providing clarity on how to affect these properties through design decisions. We found that it provides an extremely useful starting point when looking for advice on probes, and probe design in particular. Our efforts to follow the framework has produced engaging probes that have been useful to support the research inquiries of our project - the objectives of any successful probe $[4,12]$. While a few publications have described the approach taken to design particular probe tools (e.g. $[6,20])$, this framework offers detailed discussions on how design decisions affect probe properties and exemplify useful tactics. The lexicon introduced in the framework introduced a way to describe and discuss probes designs with some consistency into an otherwise ambiguous and diverse vocabulary used by different researchers/designers designing and using probes.

However, our use of this framework also revealed areas for improvement. In this section, we will discuss how this framework might be better translated, extended and improved upon. We believe that efforts towards establishing a probe design framework will be helpful especially to HCI and Interaction Design students and researchers new to designing and using probes as a tool for inquiry [21]. 
We first came across some ambiguity within the framework when we analyzed the probe examples provided by Wallace et al., in our effort to distill a more actionable guide on how to design probes. The framework refers to pace and challenge as a single probe design property and yet the examples used, described these two as separate, though related properties. For instance, a light weight icebreaker activity is shown as taking less time to complete than a more challenging task. However, when we mapped the design decisions that affect probe design properties, we found that pace and challenge are affected by different design decisions. Pace is affected by decisions such as speed, duration and frequency, while challenge is affected by decisions such as commitment and creativity levels. We therefore recommend considering these two properties as separate, as we have done so in Table 1.

When we put the framework to use, we also found it useful to think of the probe properties as something along a continuum. This was particularly useful when visualizing the three probe design properties; openness/boundedness, pace and challenge together. Boucher et al. [6] mention this continuous nature of probe properties when describing how to design engaging and productive probes; "They provide for a range of engagement...range from relatively neutral to playful." Meanwhile, Tsai et al. [20] use pairs of values to guide the design of their probes; familiaritystrangeness, definiteness-ambiguity and objective-subjective. These examples reiterate the usefulness of using continuums when conceptualizing the design properties of probes.

The importance of offering participants a diverse range of probes is widely acknowledged $[12,18,19]$ and we found that considering the set of probes along various continuums (Fig. 3) not only helps guide the design of engaging individual probes, but the strategic design of a more-balanced, varied and engaging probe collection that can more effectively steer a participant through varying levels of reflection. We made sure to include an icebreaker probe, a probe to promote deeper reflection and a probe to transition participants between these two (Fig. 2).

We found that the more discrete property of materiality is useful to consider because of its potential to offer gift-like qualities in probes we give to participants to complete. This can foster participant engagement [21]. Take for instance, how participants gravitated towards the Family Experience Jar when we unpacked our three probes. Their attention was drawn towards the stylish clear glass jar we showed them and away from the other two (paper) probes. We also realized that materiality also has the capacity to affect the properties openness/boundedness, pace and challenge. An example is Family Experience Jar. While the materiality of the jar initially engaged participants, the choice to use colourful post-it notes to break up the otherwise lengthy diary task increased pace and lowered challenge. This in turn maintained engagement throughout the study.

In their original conception by Gaver et al. [11], probes were designed with a 'spirit' of absurdity, ambiguity, mystery and

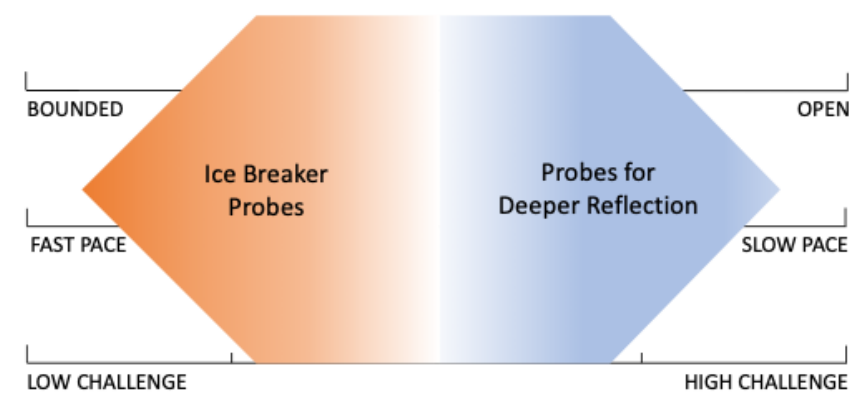

playfulness in an attempt to provoke unpredictable responses from participants $[4,12]$. Elements of this 'spirit', such as playfulness, have been carried through by researchers/designers exploring how to adapt the method to engage participants (e.g. [1, 3]). Therefore, we were surprised that the framework did not feature explicit guidance about this 'spirit'. However, given that

\section{Figure 3. Using linear scales to visualize how varying three design properties can support the design of different probe types}

the context of Wallace et al.'s work is limited to explorations of self-identity and personal significance, it is appropriate that their probe examples tend to be designed to embody sensitivity, and draw less on absurdity, mystery or playfulness etc.

While it was not explicitly mentioned in the framework, we found it necessary to look for ways to inject a sense of fun, humor and absurdity into each of our probes. For example, the lids of our Family Experience Jar were designed with a very thin opening so participants would have to fold their notes before they would fit. We also glued the lids so notes could not be removed. We anticipated the sense of secrecy, curiosity and even competition that might be introduced. As we piloted Digital Family Tree we were aware of the personal curiosity that might be arise from learning how a loved one had depicted aspects of family life. When we discussed responses to Device Journal with our participants, we found that the sense of absurdity and playfulness inherent in the design of our comic book had inspired the creativity, imagination and humour we had hoped for. We find Boucher et al.'s [6] term 'affective tone' appropriate to describe a probe design property that relates to the 'spirit' of a probe. We find that it would be useful to extend the framework by including this additional property and to explore the decisions that might affect it, beyond how neutral or playful the probe is.

Participant engagement is affected not only by how we design the probes, but also how to use them [4]. Here, we think that more guidance about how to initiate probes would be helpful such as, how to instruct participants to use our probes and how to offer support and communication while they are using them, and so on (e.g. $[18,19])$. So, a more useful framework should provide clearer guidance on the decisions involved with instructions. These might include choices on the level and format of any directions provided to explain a probe, whether to provide an example of a completed 
probe and whether to offer additional scaffolds such as sources of inspiration. Similarly, guidance on communication might include advice on whether and how to offer or require certain levels of communication with participants during the study.

In addition to the guidance from Wallace et al.'s paper, we now summarize some key points derived from our learnings. These points are some of our main contributions discussed in this paper. We hope that these ideas, when read with Wallace et al.'s framework can help extend and offer greater clarity and guidance when designing and using probes.

1. Before embarking on probe design, invest in understanding participants by holding a preliminary workshop or similar activities to get to know the participants and their situations.

2. When you are ready to design your probes, use Table 1 - our distillation and translation of the design properties, together with possible design decisions. This will support systematic considerations of the various design properties.

3. Do not think about the design properties as binary states but rather characteristics on a continuum. This will give you greater flexibility and creativity when considering your probe designs (Figure 2)

4. Consider materiality as a discrete property and consider how to use it to affect the design properties of openness/boundedness, pace and challenge.

5. Consider the additional property affective tone to help guide the design of probes that are neutral, playful, absurd etc.

6. When designing a probe collection, use the continuum of design properties to ensure that participants are offered an icebreaker probe and probes that offer varying levels of reflection.

Finally, we must acknowledge that the framework is informed by examples of probe use in which a single perspective is captured from an individual or family group. Hence, we are aware that designing our probes to capture differing perspectives held by parents within the same family introduced additional design decisions. We looked for advice within growing reports of probe use to explore families and aspects of family relationships such as intimacy [8, 9, 17]. Horst et al. [16] provide valuable insights into the challenges of designing probes with families, such as the need to cater for the diversity of individual family members (e.g. genders, ages, interests, ability, motivation etc.) as well as the need to consider privacy. Guidance such as this helped inform our additional decisions about how to design and use probes to explore differing perspectives held by parents within the same family.

We first had to decide whether to initiate probes and to review probe responses with participants on their own or together (e.g. initiating probes with participants together, reviewing probe responses with each participant on their own). In designing our probes, we had questions about whether to provide participants with individual or shared probe tools (e.g. individual Device Journals, a shared Family Experience Jar). We also had to decide whether participants' responses to our probes would be shared or private (e.g. sharing responses to Digital Family Trees, private responses to Family Experience Jar). Finally, we varied the amount of communication, comparison and collaboration permitted or required by each probe.

The framework states that probes mediate the researcherparticipant relationship. In our research project, where some probe tasks were shared between individual parent, we found that probes also mediated the relationship between these individual participants. To adapt probes to cater for the multiple perspectives that are inherent within families is not insignificant. However, as far as we are aware nobody has explicitly discussed the necessary design decisions involved in creating probes and probe activities when extending the method in this way. Emerging ubiquitous computing technologies demand that we will need to design probes that can be used productively to capture multiple perspectives within groups. Future work could provide more guidance regarding this.

\section{Towards a Probe Design Framework}

This paper presents our learnings from using Wallace et al.'s framework to guide the design and use of probes in a research inquiry. One aim is to ascertain its usefulness as a guide. Another, to see if we can contribute to clarify and extend their contribution, as well as suggesting possible future efforts that can advance us towards a more robust framework. While Wallace et al. acknowledge that their offering is "an example of what a framework for probe design and use might look like" [21] rather than a definitive guide, we would argue that efforts that can build upon their insightful work towards formulating a framework for probe design will be very useful for HCI and Interaction Design.

To be fair, we do agree with researchers who caution against being too didactic and prescriptive about how we design and use probes for fear of losing some of the creativity and designer-ly inspirations that can be seen in truly effective probes $[4,5,19]$. However, we do see the benefit of more guided reflections without being overly prescriptive. This could reduce some of the misunderstandings and misinterpretation of how probes are designed and used. At the same time, it will provide (new) researchers and designers wishing to use probes, a more robust and actionable starting point.

\section{REFERENCES}

[1] K Battarbee, A Soronen and F Mäyrä. 2004. Living in a zoo: bringing user experiences with technology to life. In Proceedings of the third Nordic conference on Human-computer interaction. ACM, 373-376.

[2] M Berkovich. 2009. Perspective Probe: Many Parts Add up to a Whole Perspective. In Proceedings of CHI '09 Extended Abstracts on Human Factors in Computing Systems. ACM, Boston, MA, USA, 2945-2954. http://dx.doi.org/10.1145/1520340.1520422

[3] R Bernhaupt, A Weiss, M Obrist and M Tscheligi. 2007. Playful Probing: Making Probing More Fun. In Proceedings of IFIP Conference on Human-Computer Interaction. Springer, 606-619.

[4] K Boehner, W Gaver and A Boucher. 2012. Probes. In Inventive Methods, Routledge, 199-215.

[5] K Boehner, J Vertesi, P Sengers and P Dourish. 2007. How HCI Interprets the Probes. In Proceedings of Proceedings of the SIGCHI Conference on Human 
Factors in Computing Systems. ACM, San Jose, California, USA, 1077-1086. http://dx.doi.org/10.1145/1240624.1240789

[6] A Boucher, D Brown, L Ovalle, A Sheen, M Vanis and W Gaver. 2018. Taskcam. In Proceedings of Extended Abstracts of the 2018 CHI Conference on Human Factors in Computing Systems. ACM, Montreal QC, Canada, 1-4. http://dx.doi.org/10.1145/3170427.3186536

[7] I Chillag. 2008. Everything is Alive. Radiotopia. Oakland, California, USA.

[8] T Dalsgaard, M B. Skov, M Stougaard and B Thomassen. 2006. Mediated Intimacy in Families: Understanding the Relation between Children and Parents. In Proceedings of Proceedings of the 2006 conference on Interaction design and children. ACM, Tampere, Finland, 145-152. http://dx.doi.org/10.1145/1139073.1139110

[9] H Davis, M.B Skov, M Stougaard and F Vetere. 2007. Virtual Box: Supporting Mediated Family Intimacy through Virtual and Physical Play. In Proceedings of Proceedings of the 19th Australasian conference on Computer-Human Interaction: Entertaining User Interfaces. ACM, Adelaide, Australia, 151-159. http://dx.doi.org/10.1145/1324892.1324920

[10] E.C Derix and T.W Leong. 2018. Days of Our Lives: Family Experiences of Digital Technology Use. In Proceedings of Proceedings of the 30th Australian Conference on Computer-Human Interaction. ACM, Melbourne, Australia, 332337. http://dx.doi.org/10.1145/3292147.3292185

[11] B Gaver, T Dunne and E Pacenti. 1999. Design: Cultural Probes. Interactions 6 , 1, 21-29. http://dx.doi.org/10.1145/291224.291235

[12] W Gaver, A Boucher, S Pennington and B Walker. 2004. Cultural Probes and the Value of Uncertainty. interactions-Funology 11, 5, 53-56.

[13] C Graham and M Rouncefield. 2008. Probes and Participation. In Proceedings of Proceedings of the Tenth Anniversary Conference on Participatory Design 2008. Indiana University, Bloomington, Indiana, 194-197.

[14] C Graham, M Rouncefield, M Gibbs, F Vetere and K Cheverst. 2007. How Probes Work. In Proceedings of Proceedings of the 19th Australasian conference on Computer-Human Interaction: Entertaining User Interfaces. ACM, Adelaide, Australia, 29-37. http://dx.doi.org/10.1145/1324892.1324899

[15] T Hemmings, A Crabtree, T Rodden, K Clarke and M Rouncefield. 2003. Probing the Probes. In Proceedings of Proc. of PDC 2002. 42-50.

[16] W Horst, T Bunt, S Wensveen and L Cherian. 2004. Designing Probes for Empathy with Families. In Proceedings of Proceedings of the conference on Dutch directions in HCI. ACM, Amsterdam, The Netherlands, 15. http://dx.doi.org/10.1145/1005220.1005239

[17] J Kjeldskov, M Gibbs, F Vetere, S Howard, S Pedell, K Mecoles and M Bunyan. 2004. Using Cultural Probes to Explore Mediated Intimacy. Australasian Journal of Information Systems 11, 2.

[18] T Mattelmäki. 2006. Design Probes. Aalto University,

[19] Interaction Research Studio. 2018. Probe Tools. Retrieved 2019 from https://probetools.net/probes.

[20] W-C Tsai, D Orth and E van den Hoven. 2017. Designing Memory Probes to Inform Dialogue. In Proceedings of Proceedings of the 2017 Conference on Designing Interactive Systems. ACM, Edinburgh, United Kingdom, 889-901. http://dx.doi.org/10.1145/3064663.3064791

[21] J Wallace, J McCarthy, P.C Wright and P Olivier. 2013. Making Design Probes Work. In Proceedings of Proceedings of the SIGCHI Conference on Human Factors in Computing Systems. ACM, Paris, France, 3441-3450. http://dx.doi.org/10.1145/2470654.2466473

[22] S.A.G Wensveen. 1999. Probing Experiences. In Proceedings of Proceedings of the first international conference on design and emotion. Delft University of Technology. Delft. 23-29. 